\title{
Two-Dimensional Spin-Polarized Hydrogen at Zero Temperature
}

\author{
L. Vranješ Markić · J. Boronat
}

Received: 13 July 2012 / Accepted: 16 September 2012 / Published online: 3 October 2012

(C) Springer Science+Business Media New York 2012

\begin{abstract}
The ground-state properties of spin polarized hydrogen $\mathrm{H} \downarrow$ in two dimensions (2D) are obtained by means of diffusion Monte Carlo calculations. Using the most accurate to date ab initio $\mathrm{H} \downarrow-\mathrm{H} \downarrow$ interatomic potential we have studied hydrogen gas phase, from the very dilute regime until densities above its freezing point. For very low densities, the equation of state of the gas can be described in terms of the gas parameter $n a^{2}$, where $a$ is the s-wave scattering length in 2D. The solid phase in $2 \mathrm{D}$ has also been studied up to high pressures and the gas-solid phase transition determined using the double-tangent Maxwell construction.
\end{abstract}

Keywords Spin-polarized hydrogen · Quantum gas · Gas-solid transition

\section{Introduction}

Spin-polarized hydrogen $(\mathrm{H} \downarrow)$ is the only system that remains in the gas phase down to the limit of zero temperature. This extreme quantum behavior is due to its low mass and extremely weak attractive part of its interatomic potential. Although it was expected to be an ideal candidate [1] for achieving the Bose-Einstein condensate (BEC) state many experimental obstacles needed to be overcome before it was finally realized in 1998 by Fried et al. [2]. Even before the realization in three dimensions (3D),

\footnotetext{
L. Vranješ Markić $(\bowtie)$

Faculty of Science, University of Split, Split 21000, Croatia

e-mail: leandra@pmfst.hr

L. Vranješ Markić

Institut für Theoretische Physik, Johannes Kepler Universität, 4040 Linz, Austria

J. Boronat

Departament de Física i Enginyeria Nuclear, Universitat Politècnica de Catalunya, 08034 Barcelona, Spain
} 
the same year, Safonov et al. [3] observed a quasicondensate in two-dimensional (2D) $\mathrm{H} \downarrow$ adsorbed on liquid ${ }^{4} \mathrm{He}$. Following experiments have been reviewed by Järvinen and Vasilyev [4], demonstrating that further work is needed to observe signals of the Berezinskii-Kosterlitz-Thouless (BKT) transition. On the other hand, the BKT transition has been observed in trapped gases of $\mathrm{Rb}$ atoms harmonically confined to move within a plane [5].

Two-dimensional Bose systems are also subject of intense theoretical interest and the study of $\mathrm{H} \downarrow$ offers the additional advantage of the accurate knowledge of the hydrogen interatomic interaction. Recently, the static fluctuation approximation was used to study finite $2 \mathrm{D} \mathrm{H} \downarrow$ in a very low-density regime [6]. The results on the condensate fraction and specific heat capacity show that the BEC occurs in the system.

In the present work, we report accurate microscopic results for energetic and structural properties of bulk $\mathrm{H} \downarrow$ in 2D. Such a study has already been completed in 3D [7] demonstrating universal behavior at low densities. At large densities, we observe that the $2 \mathrm{D}$ gas experiments a quantum phase transition to a triangular crystal.

\section{Method}

We use the diffusion Monte Carlo (DMC) method, a fully microscopic approach that solves stochastically the Schrödinger equation in imaginary time,

$$
-\hbar \frac{\partial \Psi(\boldsymbol{R}, t)}{\partial t}=\left(H-E_{t}\right) \Psi(\boldsymbol{R}, t)
$$

In Eq. (1), $E_{t}$ is a constant acting as a reference energy, while $\boldsymbol{R} \equiv\left(\boldsymbol{r}_{1}, \ldots, \boldsymbol{r}_{N}\right)$ collectively denotes particle positions. The $N$-particle Hamiltonian is given by

$$
H=-\frac{\hbar^{2}}{2 m} \sum_{i=1}^{N} \nabla_{i}^{2}+\sum_{i<j}^{N} V\left(r_{i j}\right) .
$$

$V(r)$ is the interaction potential, that has been constructed combining highly accurate $a b$ initio data for short distances [9-11] with the analytical expression for the long-range behavior, in the same way as in previous work in 3D [7]. We introduce the importance sampling wave function to guide the random walk. For the gas phase, the Jastrow model is used, $\psi_{\mathrm{J}}(\boldsymbol{R})=\prod_{i<j}^{N} f\left(r_{i j}\right)$, where $f(r)$ is the two-body correlation function. For most densities, we have chosen the functional form that was found to be optimal in 3D [7], $f(r)=\exp \left[-b_{1} \exp \left(-b_{2} r\right)\right]$, where $b_{1}$ and $b_{2}$ are variational parameters. At the lowest densities, the better form of the trial wave function was a numerical solution of the two-body problem. In the simulations of the solid phase we have used a Nosanow-Jastrow model, $\psi_{\mathrm{NJ}}(\boldsymbol{R})=\psi_{\mathrm{J}}(\boldsymbol{R}) \prod_{i}^{N} g\left(r_{i I}\right)$, where $g(r)=\exp \left(-\alpha r^{2} / 2\right)$ is a Gaussian linking every particle $i$ to its corresponding point $\mathbf{r}_{I}$ of the lattice, and $\alpha$ is a variational parameter. The variational parameters have been obtained by optimizing the variational energy. Typical values are $b_{1}=30$ and $b_{2}=0.9 \AA^{-1}$ for the liquid phase, while the optimal values in the solid phase near the melting density are $b_{1}=50, b_{2}=1.1 \AA^{-1}$ and $\alpha=0.2 \AA^{-2}$. The most relevant parameter in the solid phase $\alpha$ increases up to $\alpha=0.95 \AA^{-2}$ for the largest density studied. The influence of the time step $\Delta t$ in the DMC results is reduced by working 


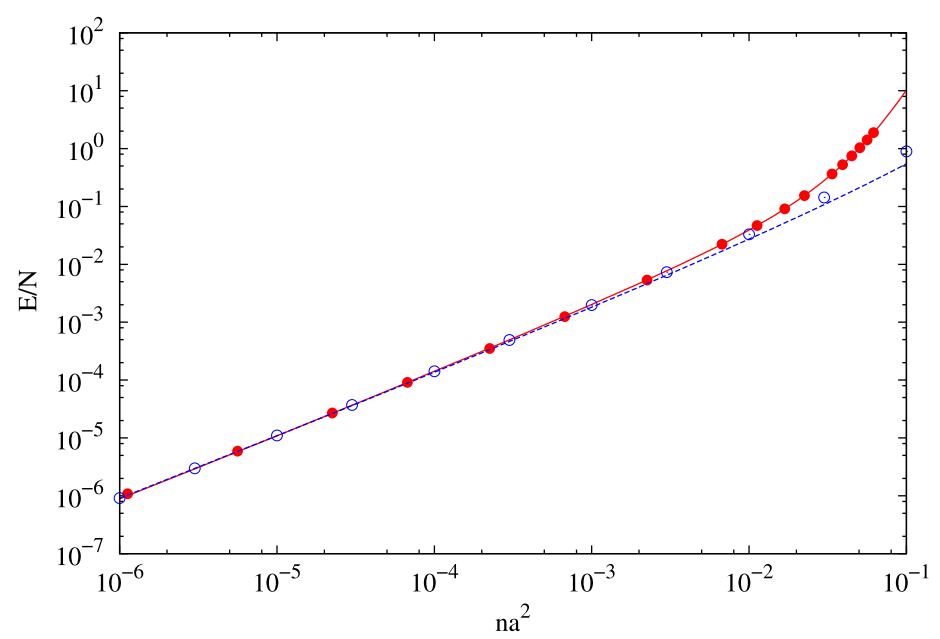

Fig. 1 Equation of state of the gas $\mathrm{H} \downarrow$ as a function of the gas parameter $n a^{2}$ in units $\hbar^{2} /\left(2 m a^{2}\right)$ (solid circles and full line). The empty circles correspond to the HS gas [12] and the line to the mean-field results (Eq. (4)) (Color figure online)

with an algorithm accurate to second order in $\Delta t[8]$. On the other hand, the size dependence due to the finite number of particles in the simulation is carefully corrected by the proper addition of asymptotic contributions. The calculations in the gas phase have been performed with 64 to 128 particles and in the solid phase with 90 to 168 particles.

\section{Results}

In Fig. 1, we plot the present DMC results for the equation of state of the gas. Our results are well parameterized by the analytical function $(e \equiv E / N)$

$$
e(\rho)=e_{1} n / \ln \left(e_{2} / n\right)+\left(e_{3} n\right)^{3}+\left(e_{4} n\right)^{5},
$$

shown as a solid line on top of the DMC results in Fig. 1. The best set of parameters is: $e_{1}=261.6(1.6) \mathrm{K} \AA^{2}, e_{2}=0.193(7) \AA^{-2}, e_{3}=48.7(4) \mathrm{K}^{-3} \AA^{2}$, and $e_{4}=28.7(2) \mathrm{K}^{-5} \AA^{2}$, the figures in parenthesis being the statistical uncertainties. On the same plot, we compare our results to hard disk energies per particle in $2 \mathrm{D}$ calculated by Pilati et al. [12] and the mean-field results given by

$$
\frac{E_{M F}}{N}=\frac{2 \pi \hbar^{2}}{m} \frac{n}{\ln \left(1 / n a^{2}\right)} .
$$

The energies per particle are plotted as a function of the gas parameter $n a^{2}$, where $a$ is the $2 \mathrm{D}$ scattering length, which we have calculated as $1.06 \AA$. We can see that the energies per particle of $\mathrm{H} \downarrow$ start to deviate from the mean-field results sooner than in the case of hard spheres. In addition, the corrections to the mean-field results can not be well fitted with the functional form appropriate for the hard sphere gas [12]. 


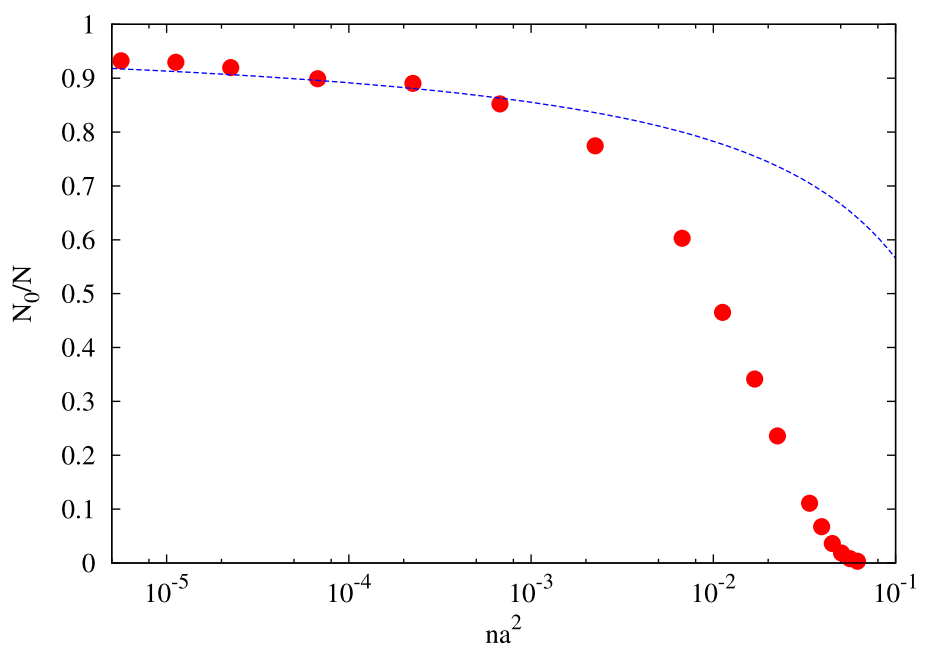

Fig. 2 Condensate fraction as a function of the gas parameter (circles). Dashed line is the result from the Bogoliubov theory (Color figure online)

The condensate fraction $N_{0} / N$, which is obtained from the long-range behavior of the one-body density matrix $N_{0} / N=\lim _{r \rightarrow \infty} \rho(r)$, presents also a universal behavior in terms of the gas parameter $n a^{2}$ at very low densities. The results, presented in Fig. 2, are compared to the Bogoliubov formula [13]

$$
\frac{N_{0}}{N}=1+\frac{1}{\ln \left(n a^{2}\right)} \text {. }
$$

For small values of the gas parameter, the results for $\mathrm{H} \downarrow$ agree with Eq. (5), while for larger $n a^{2}$ values the Bogoliubov result overestimates the condensate fraction, feature which was already observed in hard disks [12]. In accordance with results for hard disks, the condensate depletion is larger in 2D than in 3D. Using the equation of state (3), the pressure is easily derived from its thermodynamic definition $P(n)=n^{2}(\partial e / \partial n)$ and from it, the corresponding speed of sound as a function of the density, $c^{2}(n)=\frac{1}{m}\left(\frac{\partial P}{\partial n}\right)$. The pressure $P(n)$ and speed of sound $c(n)$ are shown in Fig. 3. The pressure at first rises slowly as a function of the density and then begins a rapid increase when approaching the solidification density. The speed of sound rises more slowly than in 3D. DMC also provides information on the spatial structure of the system. The results for the pair distribution function $g(r)$, obtained using pure estimators [14], are shown in Fig. 4 for five values of the gas parameter. The shell-like structure is visible for densities higher than $0.01 \AA^{-2}$.

We have also studied the solid phase of $\mathrm{H} \downarrow$ by using the Nosanow-Jastrow guiding wave function. The position of lattice sites has been selected according to a commensurate triangular phase. The results for the energy per particle are presented in Fig. 5, along with the numerical fit obtained using the function

$$
e(n)=s_{2} n^{2}+s_{3} n^{3}+s_{4} n^{4} .
$$

The optimal parameters in Eq. (6) are: $s_{2}=12470(100) \mathrm{K}^{4}, s_{3}=-2.23(4) \times$ $10^{5} \mathrm{~K} \AA^{6}$, and $s_{4}=4.08(4) \times 10^{6} \mathrm{~K}^{8}$. This expression is used to obtain the de- 


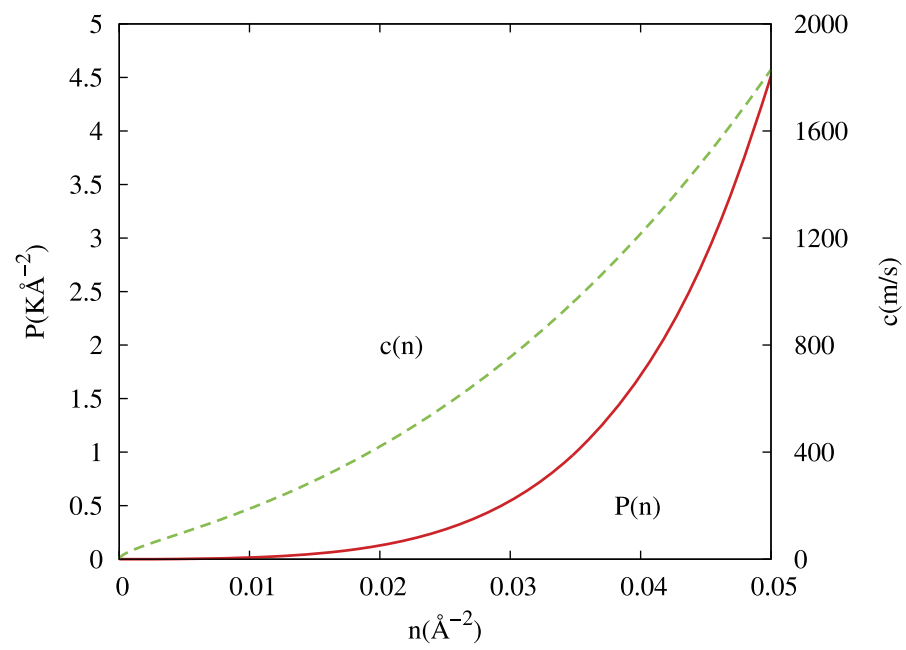

Fig. 3 Pressure and speed of sound of gas $\mathrm{H} \downarrow$ as a function of the density. Left-(right-) hand scale corresponds to pressure (speed of sound) (Color figure online)

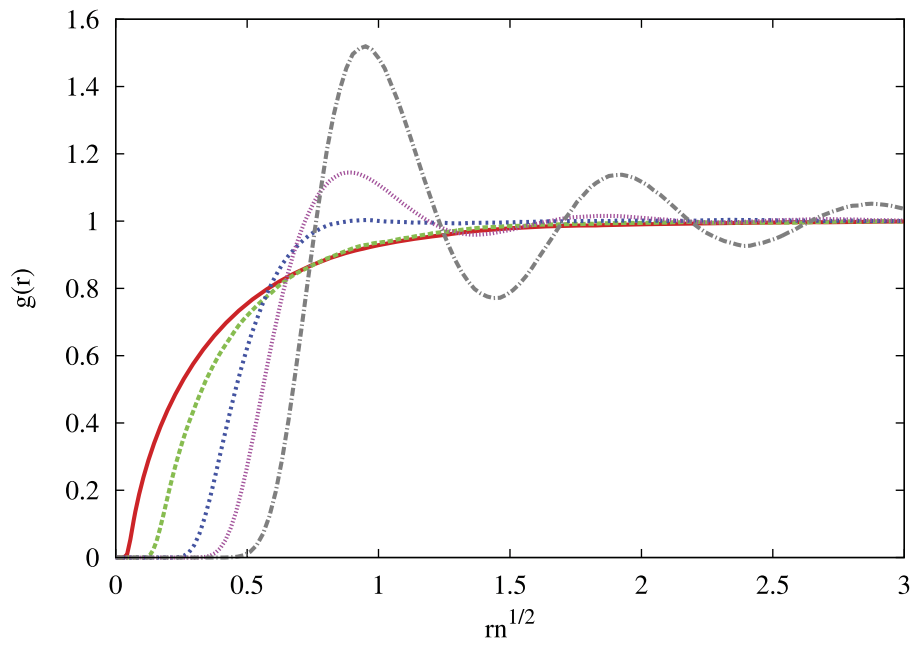

Fig. 4 Pair distribution function of $\mathrm{H} \downarrow$ for different densities, from left to right, $n=2 \times 10^{-4} \AA^{-2}$, $0.002 \AA^{-2}, 0.01 \AA^{-2}, 0.02 \AA^{-2}, 0.04 \AA^{-2}$ (Color figure online)

pendence of the pressure and the speed of sound on the density, using the same thermodynamic expressions as in the case of the gas phase. Results are presented in Fig. 5. A relevant prediction that can be drawn from the present DMC results on the energies of the gas and solid phases of $\mathrm{H} \downarrow$ in 2D is the location of the gas-solid phase transition. From the double-tangent Maxwell construction, we obtain the freezing $n_{\mathrm{f}}=0.0407 \AA^{-2}$ and melting $n_{\mathrm{m}}=0.0417 \AA^{-2}$ densities (Fig. 6). They corresponds to a common pressure at the transition of $P=1.84 \mathrm{~K}^{-2}$. Although the freezing and melting densities are very close, the spatial structure of both phases at the transition 

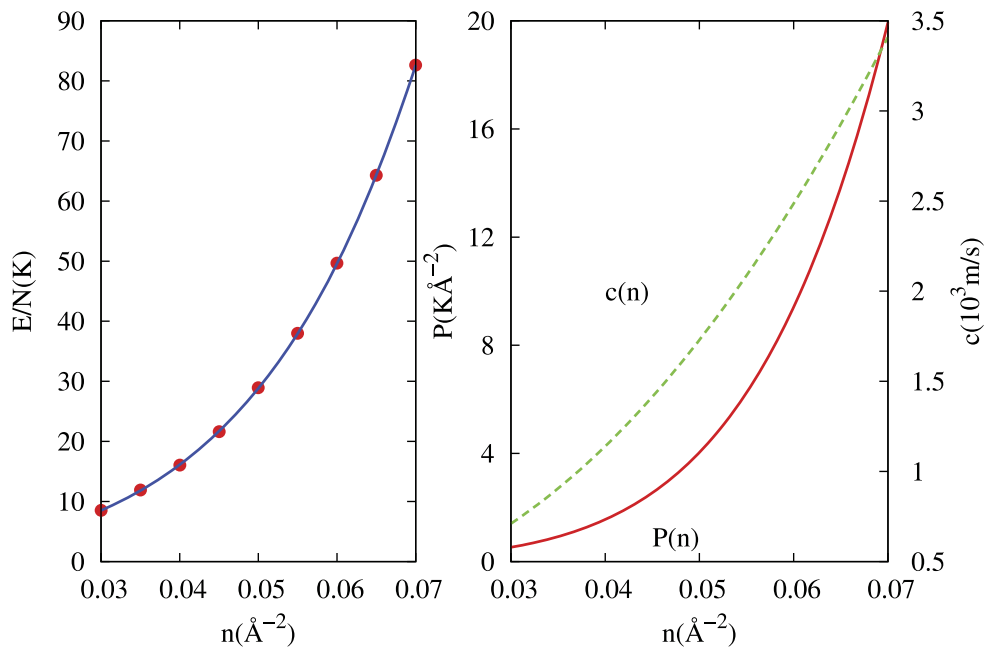

Fig. 5 Left: Energy per particle of solid $\mathrm{H} \downarrow$ as a function of the density. Right: Pressure and speed of sound of solid $\mathrm{H} \downarrow$ as a function of the density. Left-(right-) hand scale corresponds to pressure (speed of sound) (Color figure online)

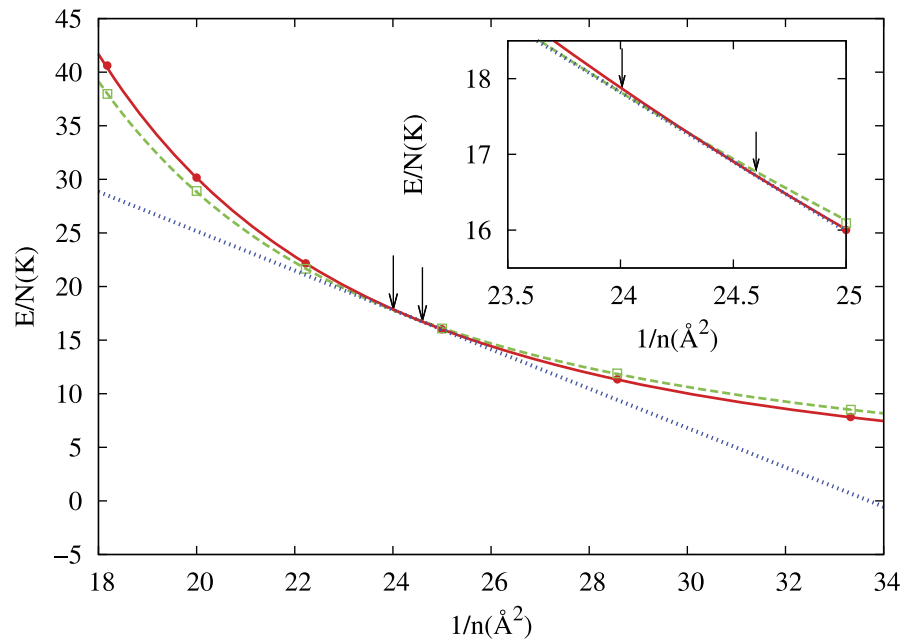

Fig. 6 Maxwell construction based on plotting the energy per particle, $E / N$ as a function of $1 / n$. The densities at which the first-order transition occurs are identified by finding the common tangent (dotted line) to both the solid (dashed line) and gas curve (full line). The inset shows the construction in a narrower range of $1 / n$ (Color figure online)

point is rather different. The difference is most visible in the static structure function shown in Fig. 7 for both phases at the transition point. High intensity Bragg peaks located at the reciprocal lattice sites provide clear signature of the solid order, which is absent in the $S(k)$ of the gas. 


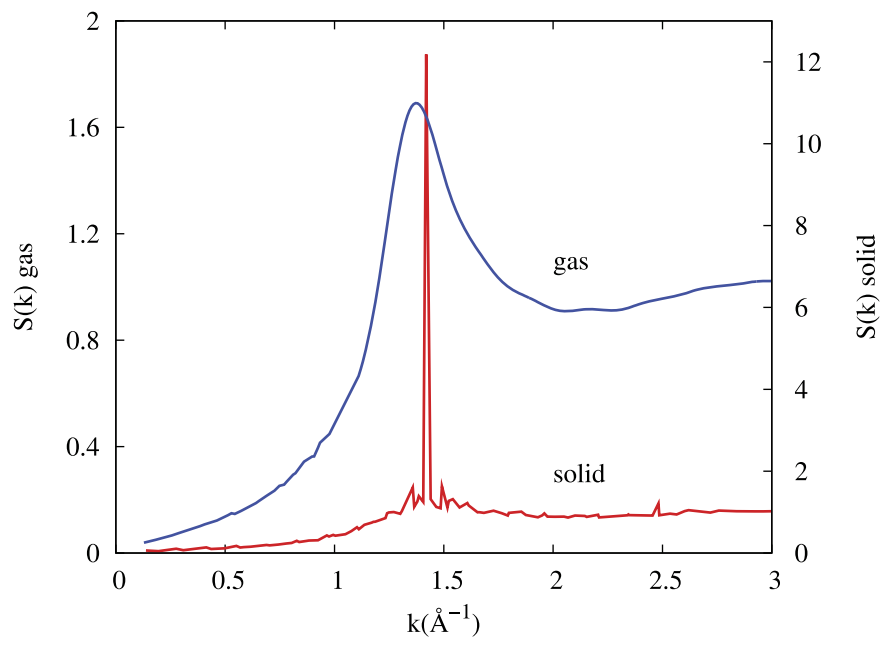

Fig. 7 Static structure factor at the gas-solid phase transition. The results correspond to the gas at $n_{f}$ and to the solid at $n_{m}$ (Color figure online)

\section{Conclusions}

We have carried out a study of the gas and solid phases of $\mathrm{H} \downarrow$ in $2 \mathrm{D}$ using the DMC method. The ground-state properties have been accurately determined using a very well-known and extremely accurate interatomic potential. In the very dilute limit, the equation of state of the gas can be described using a mean-field result for a weakly interacting Bose gas, that depends only on the gas parameter $n a^{2}$. Upon sufficient increase of the density, the system freezes. We have determined for the first time the gas-solid transition point from the equations of state of the two phases.

Acknowledgements The authors acknowledge partial financial support from MSES under Grant No. 177-1770508-0493, DGI (Spain) Grant No. FIS2011-25275 and Generalitat de Catalunya Grant No. 2009SGR-1003. We also acknowledge the support of the Central Computing Services at the Johannes Kepler University in Linz, where some of the computations were performed.

\section{References}

1. W.C. Stwaley, L.H. Nosanow, Phys. Rev. Lett. 36, 910 (1976)

2. D.G. Fried, T.C. Killian, L. Willmann, D. Landhuis, S.C. Moss, P. Kleppner, T.J. Greytak, Phys. Rev. Lett. 81, 3811 (1998)

3. A.I. Safonov, S.A. Vasilyev, I.S. Yasnikov, I.I. Lukashevich, S. Jaakkola, Phys. Rev. Lett. 81, 4545 (1998)

4. J. Järvinen, S. Vasilyev, J. Phys. Conf. Ser. 19, 186 (2005)

5. Z. Hadzibabic, P. Krueger, M. Cheneau, B. Battelier, J. Dalibard, Nature 441, 1118 (2006)

6. M.K. Al-Sugheir, H.B. Ghassib, M. Awawdeh, Phys. Rev. A 84, 013617 (2011)

7. L. Vranješ Markić, J. Boronat, J. Casulleras, Phys. Rev. B 75, 064506 (2007)

8. J. Boronat, J. Casulleras, Phys. Rev. B 49, 8920 (1994) 
9. W. Kolos, L. Wolniewicz, J. Chem. Phys. 43, 2429 (1965).

10. W. Kolos, L. Wolniewicz, Chem. Phys. Lett. 24, 457 (1974).

11. M.J. Jamieson, A. Dalgarno, L. Wolniewicz, Phys. Rev. A 61, 042705 (2000)

12. S. Pilati, J. Boronat, J. Casulleras, S. Giorgini, Phys. Rev. A 71, 023605 (2005)

13. M. Schick, Phys. Rev. A 3, 1067 (1971)

14. J. Casulleras, J. Boronat, Phys. Rev. B 52, 3654 (1995) 